\title{
Evidence for Environmentally Coupled Hydrogen Tunneling during Dihydrofolate Reductase Catalysis
}

\author{
Giovanni Maglia and Rudolf K. Allemann* \\ School of Chemistry, University of Birmingham, Edgbaston, Birmingham, B15 2TT, United Kingdom
}

Received April 17, 2003; E-mail: r.k.allemann@bham.ac.uk

The physical basis of the enormous catalytic power of enzymes is not well understood. While structural studies of enzymes have significantly influenced our view of catalysis, the static threedimensional pictures that these studies provide do not incorporate the wide range of dynamical motions that occur in proteins. Several studies have suggested that protein dynamics and enzymatic events involved in the formation and breakdown of noncovalent complexes are intimately linked. ${ }^{1-3}$ Recently a correlation between protein dynamics and bond cleavage and formation has been proposed for hydrogen transfer. ${ }^{4-13}$

Several studies of dihydrofolate reductase (DHFR) have indicated the importance of its dynamic properties for catalysis. DHFR is a ubiquitous enzyme necessary for cellular metabolism in prokaryotes and eukaryotes. It catalyses the reduction of 7,8-dihydrofolate $\left(\mathrm{H}_{2} \mathrm{~F}\right)$ using NADPH as a cofactor. In this reaction the pro- $R$ hydrogen of NADPH is transferred to C6 of the pterin substrate. ${ }^{14}$

X-ray crystallographic studies of DHFR from Escherichia coli have revealed that the enzyme adopts different conformations along the reaction pathway, depending on the bound ligand. ${ }^{3}$ NMR relaxation measurements indicated that binding of substrate and cofactor alters the dynamic properties of protein segments far from the active site, including the M20 and the FG-loops, which are known to undergo conformational changes during the reaction cycle. ${ }^{15}$ The NMR-derived order parameters and molecular dynamics simulations for various DHFR complexes have identified three main regions of motion within three surface loops. A series of sitedirected mutagenesis studies showed that the identity of the amino acid residues in these loops are important determinants of the reaction rate despite their large distance from the active site. ${ }^{16,17}$ On the basis of genomic analysis, kinetic measurements on multiple mutations, and hybrid quantum-classical molecular dynamics, a network of coupled promoting motions was recently proposed to play an important role in DHFR catalysis. ${ }^{18}$ Mixed quantum/ classical molecular dynamics simulations of the reaction catalyzed by DHFR from $E$. coli have suggested a significant tunneling contribution to the hydride-transfer rate. ${ }^{19}$ The kinetics of the NADPH-dependent reduction of $\mathrm{H}_{2} \mathrm{~F}$ catalyzed by DHFRs from several organisms have been studied extensively. ${ }^{20,21}$ To ascertain the mechanism of hydride transfer, we have investigated the temperature dependence of the kinetic isotope effect (KIE) for hydrogen transfer from the cofactor to the pterin ring of $\mathrm{H}_{2} \mathrm{~F}$ for the reaction catalyzed by DHFR from Thermotoga maritima. The physiological temperature of this thermophilic bacterium is sufficiently high that it was possible to measure the reaction rates over a relatively wide temperature range $\left(6-65^{\circ} \mathrm{C}\right)$ by fluorescence energy transfer from the protein to the reduced nicotinamide moiety of NADPH. ${ }^{22,23}$ Figure 1 indicates that the temperature dependence of the KIE over the above temperature range was biphasic with a break point at approximately $25^{\circ} \mathrm{C}$. Below $25^{\circ} \mathrm{C}$ the KIE increased with decreasing temperature, while above $25{ }^{\circ} \mathrm{C}$ the KIE was temperature independent, at least within the relatively narrow range

13372 - J. AM. CHEM. SOC. 2003, 125, 13372-13373

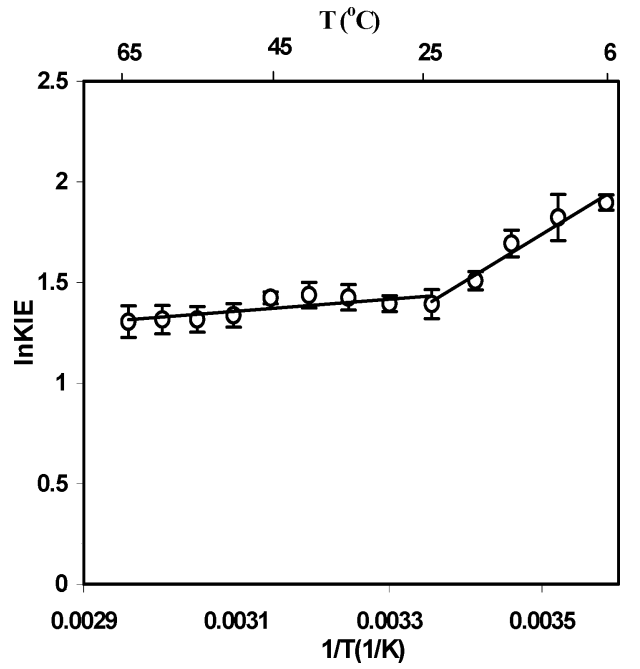

Figure 1. Kinetic isotope effects for the hydride transfer from NADPH/ NADPD to $\mathrm{H}_{2} \mathrm{~F}$ on a logarithmic abscissa versus the inverse temperature between 6 and $65^{\circ} \mathrm{C}$.

of experimentally accessible temperatures. ${ }^{22}$ A similar biphasic temperature dependence of the KIE had been observed for the reaction catalyzed by alcohol dehydrogenase from Bacillus stearothermophilus. ${ }^{9}$

The ratio of the Arrhenius preexponential factors, which was obtained from the extrapolation of the temperature dependence of the KIE, was inverse for the lower temperature range $\left(A^{\mathrm{H}} / A^{\mathrm{D}}=\right.$ $0.002 \pm 0.001)$. Above $25^{\circ} \mathrm{C}$ it fell within the semiclassical range $\left(A^{\mathrm{H}} / A^{\mathrm{D}}=1.56 \pm 0.47\right) .{ }^{24,25}$ For a reaction with no tunneling contribution, the value for $A^{\mathrm{H}} / A^{\mathrm{D}}$ is generally accepted to be close to unity. ${ }^{24}$ However, the nearly temperature-independent KIEs for the higher temperature range suggest that the hydride-transfer reaction would only be described adequately by models based on transition-state theory, if the activation energies were close to zero. ${ }^{26}$

To determine the activation energies of hydride and deuteride transfer the temperature dependence of the reaction rates was fitted to the empirical Arrhenius equation $\left[\ln k_{\mathrm{H} / \mathrm{D}}=\left(\ln \mathrm{A}^{\mathrm{H} / \mathrm{D}}\right)-\left(E_{\mathrm{A}} \mathrm{H} / \mathrm{D} /\right.\right.$ $R T$ )] for the temperature range from 25 to $65^{\circ} \mathrm{C}$ (Figure 2). The similar but relatively high values determined for $E_{\mathrm{A}}{ }^{\mathrm{H}}$ and $E_{\mathrm{A}} \mathrm{D}$ for hydride and deuteride transfer $\left(E_{\mathrm{A}}{ }^{\mathrm{H}}=53.5 \pm 0.4 \mathrm{~kJ} / \mathrm{mol} ; E_{\mathrm{A}} \mathrm{D}=\right.$ $56.0 \pm 0.8 \mathrm{~kJ} / \mathrm{mol}$ ) indicated a strong temperature dependence of the reaction rates, which together with the temperature-independent KIE suggested that the reaction occurred by vibrationally enhanced tunneling. ${ }^{9,25}$ For tunneling through a rigid barrier, the values for $E_{\mathrm{A}}{ }^{\mathrm{H}}$ and $E_{\mathrm{A}}{ }^{\mathrm{D}}$ would be expected to be close to zero, and the KIE, very large. ${ }^{27}$ However, the large activation energies and the magnitude of the KIE observed here suggested that a Marcus-like model $^{28}$ coupled to the environment best described the reaction catalyzed by DHFR from T. maritima in this temperature range. Many groups have proposed theoretical models for hydrogen 


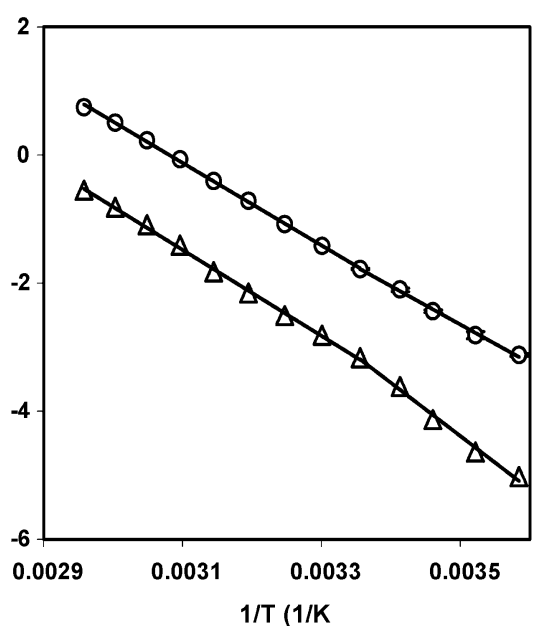

Figure 2. Arrhenius plots of kinetic data for DHFR from T. maritima (circles and triangles represent data for NADPH and NADPD, respectively).

transfer that take environmental fluctuations into account to explain the kinetic properties of several kinetic systems. ${ }^{5,11,29-33}$ Within the context of the environmentally coupled tunneling model of Knapp et al., 5,33 temperature-independent KIEs as observed here for the higher temperature range are indicative of tunneling with no (or only a small) contribution from active dynamics which actively modulate the tunneling barrier. The absence of such a gating contribution leads to almost temperature-independent tunneling distances and therefore temperature-independent KIEs. The temperature dependence of the hydrogen-transfer rates during catalysis by DHFR might stem from passive dynamics, which influence the probability of the formation of an active-site configuration from which tunneling can occur.

The biphasic behavior observed in the plots of the temperature dependence of the KIEs (Figure 1) was also seen in the Arrhenius plots (Figure 2). The slopes for deuterated and protonated substrates appeared to diverge below $25{ }^{\circ} \mathrm{C}$ due to an increased activation energy for deuteride transfer $\left(E_{\mathrm{A}} \mathrm{D}=69.2 \pm 3.7 \mathrm{~kJ} / \mathrm{mol}\right)$, while $E_{\mathrm{A}}{ }^{\mathrm{H}}$ was decreased slightly $\left(E_{\mathrm{A}}{ }^{\mathrm{H}}=49.9 \pm 1.7 \mathrm{~kJ} / \mathrm{mol}\right)$. Within a static model, where the environment does not modulate tunneling, the increased difference between the activation energies for protonated and deuterated substrates at lower temperatures together with the inverse value of $A_{\mathrm{H}} / A_{\mathrm{D}}$ suggested a reduction in tunneling. Within the environmentally coupled model for hydrogen tunneling, ${ }^{5,33}$ the kinetic parameters observed for the lower temperature range indicated a greater role for active dynamics that modulate the hydrogen-transfer distance. It is perhaps not surprising that lowfrequency protein modes become more important for the reaction at lower temperatures as they may be excited at temperatures well below the $\mathrm{C}-\mathrm{H}$ stretching mode, the excitation of which is much more temperature dependent.

The results reported herein constitute experimental support for the hypothesis that protein fluctuations during DHFR catalysis are coupled to hydride transfer via environmentally coupled quantum- mechanical tunneling. ${ }^{17,19}$ A growing number of enzymatic hydrogentransfer reactions are found to rely on quantum tunneling driven by thermally induced dynamics of the protein, suggesting a firm link between protein dynamics and enzyme catalysis.

Acknowledgment. We thank the University of Birmingham for financial support (studentship to G.M.).

Supporting Information Available: Table of the temperature dependence of single turnover rates for hydride and deuteride transfer from NADPH/D to dihydrofolate and the corresponding KIEs (PDF). This material is available free of charge via the Internet at http:// pubs.acs.org.

\section{References}

(1) Bruice, T. C.; Benkovic, S. J. Biochemistry 2000, 39, 6267-6273.

(2) Miller, G. P.; Benkovic, S. J. Chem. Biol. 1998, 5, R105-R113.

(3) Sawaya, M. R.; Kraut, J. Biochemistry 1997, 36, 586-603.

(4) Basran, J.; Patel, S.; Sutcliffe, M. J.; Scrutton, N. S. J. Biol. Chem. 2001 , 276, 6234-6242.

(5) Knapp, M. J.; Rickert, K.; Klinman, J. P. J. Am. Chem. Soc. 2002, 124 , $3865-3874$.

(6) Harris, R. J.; Meskys, R.; Sutcliffe, M. J.; Scrutton, N. S. Biochemistry 2000, 39, 1189-1198.

(7) Kohen, A.; Klinman, J. P. J. Am. Chem. Soc. 2000, 122, 10738-10739.

(8) Basran, J.; Sutcliffe, M. J.; Scrutton, N. S. Biochemistry 1999, 38, 32183222.

(9) Kohen, A.; Cannio, R.; Bartolucci, S.; Klinman, J. P. Nature 1999, 399 , 496-499.

(10) Grant, K. L.; Klinman, J. P. Biochemistry 1989, 28, 6597-6605.

(11) Antoniou, D.; Caratzoulas, S.; Kalyanaraman, C.; Mincer, J. S.; Schwartz, S. D. Eur. J. Biochem. 2002, 269, 3103-3112.

(12) Caratzoulas, S.; Mincer, J. S.; Schwartz, S. D. J. Am. Chem. Soc. 2002 124, 3270-3276.

(13) Mincer, J. S.; Schwartz, S. D. J. Phys. Chem. B 2003, 107, 366-371.

(14) Charlton, P. A.; Young, D. W.; Birsall, B.; Feeney, J.; Roberts, G. C. K J. Chem. Soc., Chem. Commun. 1979, 922-924.

(15) Osborne, M. J.; Schnell, J.; Benkovic, S. J.; Dyson, H. J.; Wright, P. E. Biochemistry 2001, 40, 9846-9859.

(16) Cameron, C. E.; Benkovic, S. J. Biochemistry 1997, 36, 15792-15800.

(17) Rajagopalan, P. T. R.; Lutz, S.; Benkovic, S. J. Biochemistry 2002, 41 $12618-12628$

(18) Agarwal, P. K.; Billeter, S. R.; Rajagopalan, P. T. R.; Benkovic, S. J Hammes-Schiffer, S. Proc. Natl. Acad. Sci. U.S.A. 2002, 99, 2794-2799.

(19) Agarwal, P. K.; Billeter, S. R.; Hammes-Schiffer, S. J. Phys. Chem. B 2002, 106, 3283-3293.

(20) Appleman, J. R.; Beard, W. A.; Delcamp, T. J.; Prendergast, N. J.; Freisheim, J. H.; Blakley, R. L. J. Biol Chem 1990, 265, 2740-2448.

(21) Fierke, C. A.; Johnson, K. A.; Benkovic, S. J. Biochemistry 1987, 26, 4085-4092.

(22) Experimental details of the stopped-flow kinetic measurement and the rate constants for hydride/deuteride transfer and the KIEs are given in Supporting Information.

(23) $\left[(4-R)-{ }^{2} \mathrm{H}\right]-\mathrm{NADPH}(\mathrm{NADPD})$ was prepared as previously described: Maglia, G.; Javed, M. H.; Allemann, R. K. Biochem. J. 2003, 374, 529535 .

(24) Bell, R. P. The Tunneling Effect in Chemistry; Chapman \& Hall: London, 1980.

(25) Melander, L.; Saunders, W. H. Reaction Rates of Isotopic Molecules; Krieger: Malabar, FL, 1987.

(26) Jonsson, T.; Glickman, M. H.; Sun, S. J.; Klinman, J. P. J. Am. Chem Soc. 1996, 118, 10319-10320.

(27) Benderskii, V. A.; Makarov, D. E.; Wight, C. A. Adv. Chem. Phys. 1994, $88,151-207$.

(28) Marcus, R. A.; Sutin, N. Biochim. Biophys. Acta 1985, 811, 265-322.

(29) Borgis, D. C.; Lee, S. Y.; Hynes, J. T. Chem. Phys. Lett. 1989, 162, 1926.

(30) Borgis, D. C.; Hynes, J. T. Chem. Phys. 1993, 170, 315-346.

(31) Borgis, D. C.; Hynes, J. T. J. Phys. Chem. 1996, 100, 1118-1128.

(32) Bruno, W. J.; Bialek, W. Biophys. J. 1992, 63, 689-699.

(33) Knapp, M. J.; Klinman, J. P. Eur. J. Biochem. 2002, 269, 3113-3121. JA035692G 